\title{
Hydrogen isotopic effects on the chemical erosion of graphite induced by ion irradiation
}

\author{
J.H. Liang ${ }^{(a)}$, M. Mayer ${ }^{(b)}$, J. Roth ${ }^{(b)}$, M. Balden ${ }^{(b)}$, W. Eckstein ${ }^{(b)}$ \\ a National Tsing Hua University, Department of Engineering and System Science, \\ Hsinchu 300, Taiwan, R.O.C. \\ b Max-Planck-Institute fuer Plasmaphysik, Boltzmannstrasse 2, D-85748 Garching, \\ Germany
}

\begin{abstract}
This theoretical study investigates the dynamic behavior of chemical erosion of graphite due to hydrogen-isotope-ion bombardment. Ion energies range from 10 to $1000 \mathrm{eV}$ and target temperatures range from 300 to $1100 \mathrm{~K}$. The computer code employed was TRIDYN. The chemical erosion processes under investigation included surface-related and thermally activated hydrocarbon emission processes. The proposed simulation model of this study was fitted to actual measurements by implementing surface-related and thermally activated coefficients. In addition, the proposed simulation model of this study was improved over our previous model by incorporating a depth-dependent probability for out-diffusion of hydrocarbons. The local reduction of carbon density due to either physical sputtering or chemical erosion was also taken into consideration. Furthermore, application of the proposed simulation model of this study was extended to include all hydrogen isotope ions (i.e. hydrogen, deuterium, and tritium). It was found that all the calculated, fitted, and measured results are in good agreement. In particular, the results calculated from the proposed simulation model of this study surpass previous ones in the low ion energy region in which chemical erosion is of vital importance.
\end{abstract}

PACS number: $\quad$ 81.05.Uw; 25.60.Pj; 92.40.Gc; 32.10.Bi; 68.49.Sf

Keywords: Graphite; Thermonuclear fusion; Chemical erosion; Hydrogen isotope; Physical sputtering 


\section{Introduction}

In recent decades, the suitability of graphite as an inner-wall material in thermonuclear fusion devices $[1,2]$ has attracted considerable interest in both theoretical simulations and experimental measurements mainly due to its superior thermal and mechanical properties. However, in addition to physical sputtering, the erosion of graphite can be greatly enhanced by chemical release of gaseous hydrocarbons (so-called chemical erosion) via chemical reaction between hydrogen isotope atoms and carbon atoms through surface-related and thermally activated hydrocarbon emission processes [3]. In essence, the hydrocarbons consist of predominantly methane molecules with smaller contributions of higher hydrocarbons [4]. Surface-related process refers to ion induced release of weakly bound hydrocarbon surface complexes while thermally activated process refers to thermal release of hydrocarbons at the end of ion range. Chemical erosion processes result in undesirable effects such as a limited lifetime of components, plasma dilution due to impurities, and high tritium retention in redeposited layers [3]. Hence, a thorough understanding of the interaction of graphite with hydrogen isotope ions $(\mathrm{H}$, $\mathrm{D}$, and $\mathrm{T}$ ) is essential before considering thermonuclear fusion for industrial applications. Additionally, a comprehensive model for simulating the chemical erosion of graphite under bombardment of hydrogen isotope ions is necessary in order to gain more insight in chemical erosion mechanisms and in designing more suitable inner-wall materials (such as doped graphite [5]). It is therefore the objective of this study to improve upon our previous model [6] by incorporating it with the diffusion model proposed by Hopf et al. [7] as well as extending its applicability to all hydrogen isotope ions. Both chemical erosion and physical sputtering behaviors of graphite in responding to various ion energies $(E)$ and target temperatures $(T)$ are also studied in depth.

\section{Simulation model}


The total erosion yield $\left(Y_{\text {tot }}\right)$ of graphite under hydrogen isotope ion irradiation is given by the following widely-accepted equation [3]:

$$
Y_{\text {tot }}=Y_{\text {phys }}+Y_{\text {chem }}=Y_{\text {phys }}+Y_{\text {surf }}+Y_{\text {therm }},
$$

where $Y_{\text {phys }}$ denotes the physical sputtering yield of graphite due to the emission of carbon atoms from the target surface, $Y_{\text {chem }}$ represents the chemical erosion yield of graphite due to the emission of hydrocarbons, $Y_{\text {surf }}$ denotes the chemical erosion yield of graphite due to the emission of surface-related hydrocarbons from the target surface, and $Y_{\text {therm }}$ represents the chemical erosion yield of graphite due to the emission of thermally activated hydrocarbons from both the target surface and bulk.

In this study, the proposed simulation model of $Y_{\text {surf }}$ and $Y_{\text {therm }}$ is improved upon our previous one [6] by including an out-diffusion probability of hydrocarbons [7]. That is, the calculated surface-related and thermally activated chemical erosion yields are given by the fluence-averaged values as follows:

$$
\begin{array}{ll}
Y_{\text {surf }}^{\text {calc }}=\frac{1}{\Phi} \sum_{i} \alpha \frac{(\mathrm{H} / \mathrm{C})_{x_{i}}}{(\mathrm{H} / \mathrm{C})_{\max }} f_{d, x_{i}} P_{\text {diff, } x_{i}} & \text { if } 0 \leq x_{i} \leq x_{\text {suf }} \\
Y_{\text {therm }}^{\text {calc }}=\frac{1}{\Phi} \sum_{i} \beta \frac{(\mathrm{H} / \mathrm{C})_{x_{i}}}{(\mathrm{H} / \mathrm{C})_{\max }} Y_{\text {therm }}^{\text {meas }} & \text { if } 0 \leq x_{i},
\end{array}
$$

where the superscripts "calc" and "meas" respectively denote the calculated values yielded from this study and the measured data obtained from other studies; the subscripts "surf" and the "therm" respectively represent surface-related and thermally activated processes in emitting hydrocarbons; and the subscript " $i$ " denotes the $i$-th implanted hydrogen isotope ion. In addition, (i) $\Phi=\phi t$ denotes the hydrogen isotope ion fluence, $\phi$ represents the hydrogen isotope ion flux, and $t$ denotes implantation time; (ii) $\alpha$ and $\beta$ respectively represent the surface-related and thermally activated coefficients that are to be determined; (iii) $(\mathrm{H} / \mathrm{C})_{x_{i}}$ refers to the atomic ratio of hydrogen isotope to carbon at the location $x_{i}$ where it attempts to saturate to a temperature-dependent value $(\mathrm{H} / \mathrm{C})_{\max }$ as ion fluence exceeds some critical fluence $\Phi_{\text {crit }}$; (iv) $(\mathrm{H} / \mathrm{C})_{\max }$ decreases exponentially 
from $0.42[8,9]$ to approximately 0.0 as target temperature increases from 300 to $1100 \mathrm{~K}$ and is herein assumed to be identical for all hydrogen isotopes; (v) $x_{i}$ indicates the location or depth of the $i$-th implanted hydrogen isotope ion in graphite [10]; (vi) $f_{d, x_{i}}$ refers to implantation damage produced at $x_{i}$ due to the $i$-th implanted hydrogen isotope ion $[3,10,11]$; and (vii) $P_{\text {diff, } x_{i}}=e^{-x_{i} / \lambda}$ refers to the depth-dependent probability of out-diffusing hydrocarbons from $x_{i}$ due to the $i$-th implanted hydrogen isotope ion [7]. $\lambda$ denotes diffusion length and is assumed to increase linearly with target temperature (i.e. $\lambda=0.4 \mathrm{~nm}$ at $300 \mathrm{~K}$ [7] and $\lambda=x_{\text {surf }}$ at $1100 \mathrm{~K}$ ); and $x_{\text {surf }}$ represents the maximum depth at which chemical erosion of graphite from the target surface takes place and is given by $1 \mathrm{~nm}$ [12]. The possibility of segregation and formation of gas molecules such as $\mathrm{H}_{2}, \mathrm{D}_{2}$, or $T_{2}[13]$ in graphite is neglected in the present study.

Furthermore, in this study, chemical erosion of graphite due to hydrogen isotope ion irradiation was dynamically conducted by implementing Eqs. (2) and (3) in the TRIDYN [14] dynamic Monte-Carlo computer simulation code. The ion fluence and ion flux employed were $1.25 \times 10^{19} \mathrm{ions} / \mathrm{cm}^{2}$ and $10^{16} \mathrm{ions} / \mathrm{cm}^{2} \mathrm{~s}$, respectively [15]. The number of simulation particles used in all computations is $10^{6}$. In addition, an iteration procedure as well as the least-squares fitting technique were applied to best approximate $Y_{\text {surf, therm }}^{\text {calc }} Y_{\text {surf, therm }}^{\text {meas }}$ so that the best-fitting coefficients $\alpha$ and $\beta$ could be determined. Notably, Roth's [3] fit formula of $Y_{\text {surf, therm }}^{\text {Roth }}$ approximates well to $Y_{\text {surf, therm }}^{\text {meas }}$ and is thus adopted in the present study to represent $Y_{\text {surf, therm }}^{\text {meas }}$ whenever needed.

\section{Results and discussion}

Table 1 tabulates the best-fitting coefficients $\alpha$ and $\beta$ obtained from this study for graphite bombarded by hydrogen isotope ions at various target temperatures. As can be seen, $\alpha$ is more dependent on target temperature than is 
$\beta$. In addition, $\alpha$ decreases as target temperature increases. Conversely, $\beta$ increases moderately as target temperature increases, peaks at $773 \mathrm{~K}$, and then decreases as target temperature increases further. Also, $\mathrm{D}$ and $\mathrm{H}$ ions hold the largest $\alpha$ and $\beta$, respectively, among all of the hydrogen isotope ions. The former indicates that $\mathrm{D}$ possesses the greatest ability to form gaseous hydrocarbons [16] while the latter reveals that the largest amount of $\mathrm{H}$ ions are reflected from the target surface and end up at a depth that isn't saturated with the $(\mathrm{H} / \mathrm{C})_{\max }$ value [6].

In essence, $Y_{\text {surf }}, Y_{\text {therm }}$, and $Y_{\text {phys }}$ values increase with increasing ion mass. Take D ion irradiation, for example. The dependence of $Y$ on ion energy due to $\mathrm{D}$ ion irradiation at target temperatures of 300 and $773 \mathrm{~K}$ is plotted in Fig. 1. In the figure, the calculated results from this study $\left(Y^{\text {calc }}\right)$, the calculated results from previous study ( $\left.Y^{\text {prev }}\right)$ [6], and the fitted results from Roth's fit formula [3] ( $Y^{\text {Roth }}$ ) are illustrated by solid, dashed, and dotted lines, respectively. As can be seen, the surface-related and thermally activated chemical erosion processes are dominant at low and high target temperatures, respectively. Comparing $Y^{\text {calc }}$ with $Y^{\text {prev }}$ reveals that all of the $Y_{\text {surf }}^{\text {calc }}, Y_{\text {therm }}^{\text {calc }}$, and $Y_{\text {phys }}^{\text {calc }}$ values follow the corresponding $Y^{\text {prev }}$ sufficiently well. However, there are some discrepancies between $Y_{\text {surf }}^{\text {calc }}$ and $Y_{\text {surf }}^{\text {prev }}$ especially in the low ion energy region $(<40 \mathrm{eV})$ and will be discussed later. In addition, all of the $Y_{\text {surf }}^{\text {calc }}, Y_{\text {therm }}^{\text {calc }}$, and $Y_{\text {phys }}^{\text {calc }}$ values reproduce the corresponding $Y^{\text {Roth }}$ values quite well. However, there are some discrepancies between $Y_{\text {phys }}^{\text {calc }}$ and $Y_{\text {phys }}^{\text {Roth }}$, primarily due to that fact that Roth's fit formula for $Y_{\text {phys }}$ is derived with the underlying assumption that the graphite target material does not change its $\mathrm{C}$ concentration during $\mathrm{D}$ ion bombardment. In reality, $\mathrm{D}$ ion irradiation causes a reduction of $\mathrm{C}$ fraction in the target material, subsequently resulting in a decrease of $Y_{\text {phys }}^{\text {calc }}$ when compared to $Y_{\text {phys }}^{\text {Roth }}$. In addition, $(D / C)_{\max }$ increases (i.e. the atomic fraction of carbon decreases) as target temperature decreases. Hence, the difference between $Y_{\text {phys }}^{\text {calc }}$ and $Y_{\text {phys }}^{\text {Roth }}$ widens as target 
temperature decreases. Similar phenomena occur in both $H$ and $T$ ion bombardments. Also notice that the discrepancy between $Y_{\text {phys }}^{\text {calc }}$ and $Y_{\text {phys }}^{\text {Roth }}$ increases as ions become heavier.

It is worth noting that chemical erosion significantly modifies $\mathrm{C}$ concentration at the target surface. Consequently, the threshold ion energy that initiates physical sputtering $\left(E_{\text {thres }}\right)$ changes with target temperature as well as hydrogen isotope. In this study, $E_{\text {thres }}$ is the ion energy at which $Y_{\text {phys }}$ equals $10^{-4}$. The values of $E_{\text {thres }}^{\text {Roth }}$ are $40.0,32.2$, and $34.2 \mathrm{eV}$ for $\mathrm{H}, \mathrm{D}$, and $\mathrm{T}$ ion irradiations, respectively. These values are compared to those of $E_{\text {thres }}^{\text {calc }}$ at target temperatures of 300 and 773 $\mathrm{K}: 43.2$ and $44.1 \mathrm{eV}$ for $\mathrm{H}$ ion irradiation; 28.1 and $29.2 \mathrm{eV}$ for $\mathrm{D}$ ion irradiation; and 23.1 and $26.6 \mathrm{eV}$ for $\mathrm{T}$ ion irradiation. That is, $E_{\text {thres }}^{\text {Roth }}$ is independent of target temperature, but retains its maximum value for $\mathrm{H}$ ion irradiation and its minimum

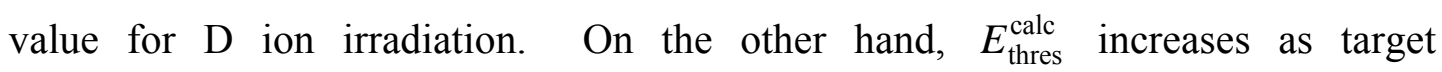
temperature increases but decreases as ion mass increases.

Fig. 2 displays $Y_{\text {tot }}$ as a function of ion energy due to $\mathrm{D}$ ion irradiation at target temperatures of 300 and $773 \mathrm{~K}$, in which $Y_{\text {tot }}^{\text {calc }}, Y_{\text {tot }}^{\text {prev }}, Y_{\text {tot }}^{\text {Roth }}$, and $Y_{\text {tot }}^{\text {meas }}$ $[17,18]$ are shown by solid line, dashed line, dotted line, and open square, respectively. Basically, the results indicate that $Y_{\text {tot }}^{\text {calc }}$ at both target temperatures are consistent with the corresponding $Y_{\text {tot }}^{\text {Roth }}$ as well as $Y_{\text {tot }}^{\text {meas }}$. At a target temperature of $300 \mathrm{~K}, Y_{\text {tot }}^{\text {calc }}$ with a low ion energy corresponds closer to $Y_{\text {tot }}^{\text {meas }}$ when compared to both $Y_{\text {tot }}^{\text {prev }}$ and $Y_{\text {tot }}^{\text {Roth }}$ versus $Y_{\text {tot }}^{\text {meas }}$. This is one of the major achievements of the simulation model proposed in this study. Furthermore, the fact that when $Y_{\text {phys }}^{\text {calc }}$ is at a high ion energy, it is smaller than that of $Y_{\text {phys }}^{\text {Roth }}$ (as shown in Figure 1), accounting for why $Y_{\text {tot }}^{\text {calc }}$ is smaller than $Y_{\text {tot }}^{\text {Roth }}$.

In Fig. 3, $Y_{\text {chem }}$ versus target temperature due to 50 and $100 \mathrm{eV} \mathrm{D}$ ion irradiation at target temperatures of 300 and $773 \mathrm{~K}$ is displayed by a comparison of $Y_{\text {chem }}^{\text {calc }}, Y_{\text {chem }}^{\text {prev }}, Y_{\text {chem }}^{\text {Roth }}$, and $Y_{\text {chem }}^{\text {meas }}$. As can be seen, all of the $Y_{\text {chem }}^{\text {calc }}, Y_{\text {chem }}^{\text {prev }}, Y_{\text {chem }}^{\text {Roth }}$, 
and $Y_{\text {chem }}^{\text {meas }}$ values correspond closely to each other. In addition, $Y_{\text {chem }}$ exhibits a peak at high target temperatures for ion energies of 50 and $100 \mathrm{eV}$. This is due to the thermally activated process and is detailed in Refs. [19-21]. Peak target temperature $\left(T_{\text {chem }}^{\text {peak }}\right)$ is the temperature at which $Y_{\text {chem }}$ reaches its maximum. The

values of $T_{\text {chem }}^{\text {peak }}$ at ion energies of 50 and $100 \mathrm{eV}$ are, respectively, 820 and $821 \mathrm{~K}$ for $\mathrm{H}$ ion irradiation; 817 and $820 \mathrm{~K}$ for D ion irradiation; and 816 and $819 \mathrm{~K}$ for $\mathrm{T}$ ion irradiation. That is, $T_{\text {chem }}^{\text {peak }}$ decreases slightly with heavier ions and smaller ion energies.

\section{Conclusions}

This study has successfully developed a comprehensive computer model to simulate chemical erosion of graphite due to hydrogen isotope ion irradiation. The calculated results correlate more closely to the measured data than do the fitted results yielded by Roth. Both physical sputtering and chemical erosion yields vary with ion energy, target temperature, and ion mass. In particular, the hydrogen isotopic effect has influence on: (1) the surface-related coefficient; (2) the thermally activated coefficient; (3) the threshold ion energy for physical sputtering; and (4) the target temperature at which chemical erosion yield reaches its maximum. Among them, the surface-related coefficient displays stronger target-temperature dependence than does the thermally activated coefficient. Deuterium and hydrogen ions hold the largest surface-related and thermally activated coefficients, respectively, among all of the hydrogen isotope ions. The threshold ion energy for physical sputtering increases with higher target temperature, but decreases with greater ion mass. The target temperature at which chemical erosion yield reaches its peak increases with increasing ion energy but decreases with greater ion mass.

\section{Acknowledgments}

The authors would like to thank Dr. W. Jacob for stimulating many valuable 
discussions. This study was financially supported by the Max-Planck-Institute for Plasmaphysik (Federal Republic of Germany) and the National Science Council (Republic of China).

\section{References}

[1] J. Roth and C. García-Rosales, Nucl. Fusion 36 (1996) 1647 with corrigendum Nucl. Fusion 37 (1997) 897.

[2] R. Parker, G. Janeschitz, H.D. Pacher, D. Post, S. Chiocchio, G. Federici, P. Ladd, ITER Joint Central Team, Home Teams, J. Nucl. Mater. 241-243 (1997) 1 .

[3] J. Roth, J. Nucl. Mater. 266-269 (1999) 51.

[4] E. Vietzke, K. Flaskamp, V. Philipps, G. Esser, P. Wienhold, J. Winter, J. Nucl. Mater. 145-147 (1987) 443.

[5] R. Schwörer, H. Plank, J. Roth, J. Nucl. Mater. 241-243 (1997) 1156.

[6] J.H. Liang, M. Mayer, J. Roth, W. Eckstein, Nucl. Instr. and Meth. B 202 (2003) 195.

[7] C. Hopf, A. von Keudell, W. Jacob, J. Appl. Phys. 94 (2003) 2373.

[8] D.K Brice, B.L. Doyle, W.R. Wampler, J. Nucl. Mater. 111\&112 (1982) 598. W. Möller, B.M.U. Scherzer, J. Appl. Phys. 64 (1988) 4860.

[9] W. Möller, J. Nucl. Mater. 162-164 (1989) 138.

[10] W. Möller, B.M.U. Scherzer, Appl. Phys. Lett. 50 (1987) 1870.

[11] J. Roth, J. Bohdansky, N. Poschenrieder, M.K. Sinha, J. Nucl. Mater. 63 (1976) 222.

[12] C. García-Rosales and J. Roth, J. Nucl. Mater. 196-198 (1992) 573.

[13] M. Balooch, D.R. Olander, J. Chem. Phys. 63 (1975) 4772.

[14] W. Möller, W. Eckstein, J.P. Biersack, Comput. Phys. Commun. 51 (1988) 355.

[15] J. Roth and C. García-Rosales, Nucl. Fusion 36 (1996) 1647 with corrigendum Nucl. Fusion 37 (1997) 897.

[16] P.W. Atkins, "Physical Chemistry," Fifth edition, Oxford University Press, Oxford, UK, 1994.

[17] M. Balden, J. Roth, J. Nucl. Mater. 280 (2000) 39.

[18] M. Balden, private communication (2004).

[19] A. Horn, A. Schenk, J. Biener, B. Winter, C. Lutterloh, M. Wittmann, J. Küppers, Chem. Phys. Lett. 231 (1994) 193.

[20] J. Biener, U.A. Schubert, A. Schenk, B. Winter, G. Lutterloh, J. Küppers, J. Chem. Phys. 99 (1993) 3125.

[21] A. Schenk, B. Winter, J. Biener, G. Lutterloh, U.A. Schubert, J. Küppers, J. Appl. Phys. 77 (1995) 2462. 
Table 1. Best-fitting coefficients $\alpha$ and $\beta$ for graphite bombarded by hydrogen isotope ions at various target temperatures.

\begin{tabular}{ccc}
\hline$T(\mathrm{~K})$ & $\alpha^{(1)}$ & $\beta$ \\
\hline 300 & $0.0500^{(2)} / 0.0714^{(3)} / 0.0593^{(4)}$ & $1.42 / 1.31 / 1.23$ \\
473 & $0.0378 / 0.0545 / 0.0451$ & $1.44 / 1.32 / 1.24$ \\
573 & $0.0356 / 0.0503 / 0.0410$ & $1.46 / 1.33 / 1.24$ \\
673 & $0.0365 / 0.0486 / 0.0387$ & $1.51 / 1.35 / 1.25$ \\
773 & $0.0366 / 0.0464 / 0.0364$ & $1.54 / 1.37 / 1.27$ \\
873 & $0.0264 / 0.0330 / 0.0257$ & $1.49 / 1.33 / 1.23$ \\
$973^{(5)}$ & $0.00741 / 0.00926 / 0.00722$ & $1.48 / 1.32 / 1.22$ \\
$1100^{(5)}$ & $0.000264 / 0.000330 / 0.000258$ & $1.47 / 1.32 / 1.22$ \\
\hline \hline Suggested & $0.0372 / 0.00507 / 0.0410$ & $1.48 / 1.31 / 1.24$ \\
\hline
\end{tabular}

(1) Calculated according to the number of hydrocarbons produced per eV /angstrom.

(2) $\mathrm{H}$ into $\mathrm{C}$

(3) D into $\mathrm{C}$

(4) $\mathrm{T}$ into $\mathrm{C}$

(5) $(\mathrm{H} / \mathrm{C})_{\max }$ was assumed to be $10^{-4}$ for target temperatures greater than $900 \mathrm{~K}$.

(6) Suggested value by averaging the values from 300 to $873 \mathrm{~K}$. 


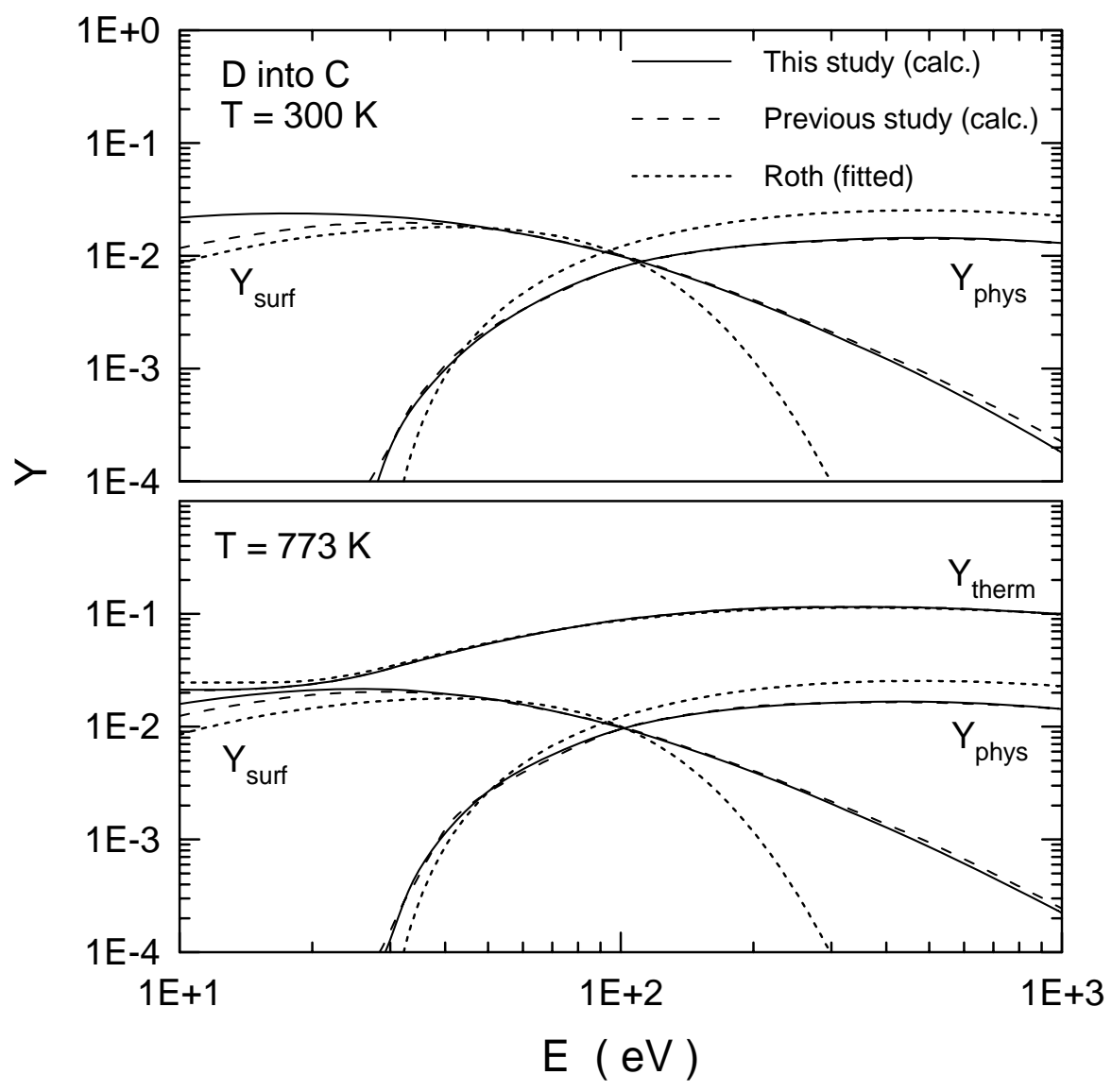

Figure 1. $Y_{\text {surf }}, Y_{\text {therm }}$, and $Y_{\text {phys }}$ as a function of ion energy for graphite bombarded by deuterium ions at target temperatures of 300 and $773 \mathrm{~K}$. 


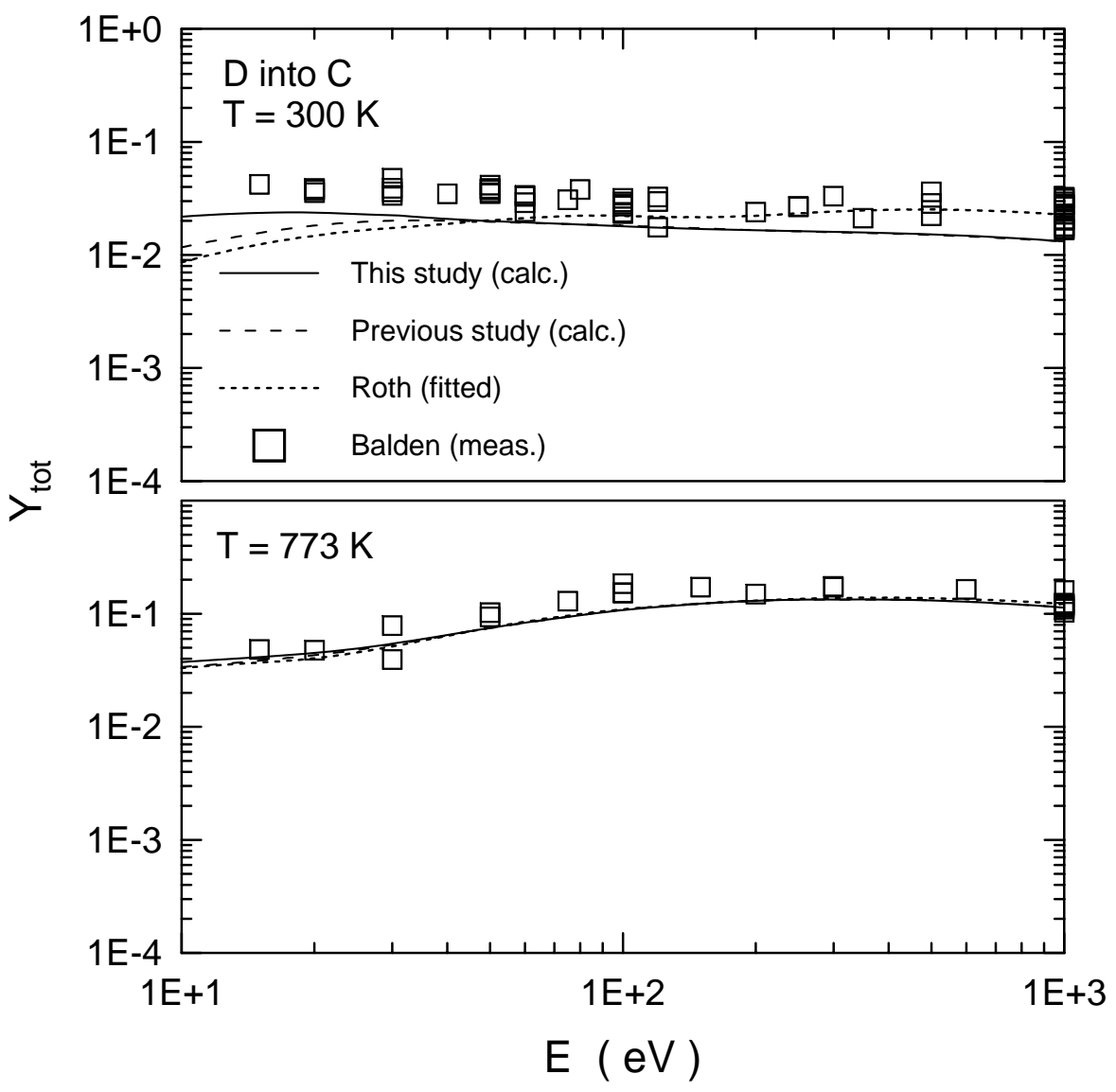

Figure 2. $Y_{\text {tot }}$ as a function of ion energy for graphite bombarded by deuterium ions at target temperatures of 300 and $773 \mathrm{~K}$. 


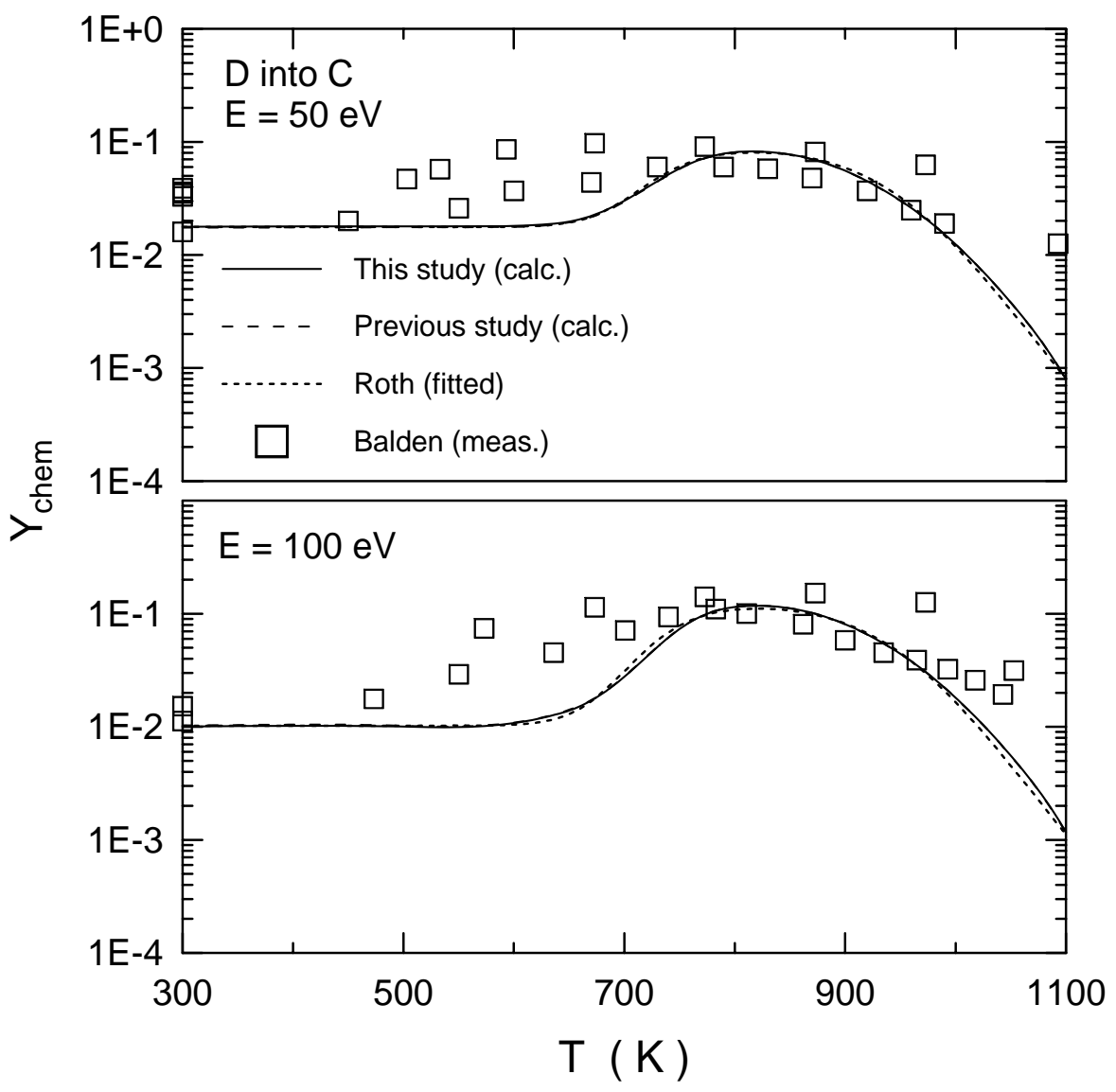

Figure 3. $\quad Y_{\text {chem }}$ as a function of target temperature for graphite bombarded by deuterium ions at ion energies of 50 and $100 \mathrm{eV}$. 